\title{
Hay (algo de) verdad en eso
}

\section{(1) Camillo Fiore}

Universidad de Buenos Aires, Argentina.

Recibido el 20/1/2018; aceptado el 5/3/2018.

\begin{abstract}
Resumen
En este trabajo presento un tratamiento formal para la expresión "hay (algo de) verdad en eso". Adopto un lenguaje de primer orden y asumo una interpretación bivaluada. Sostengo que "hay (algo de) verdad en $x$ " se comporta como una atribución de verdad parcial y no transparente. Argumento que debe ser modelada utilizando un predicado y no un operador. Introduzco un predicado y considero tres criterios alternativos para caracterizar su semántica. Pruebo que, con cualquiera de los criterios, el predicado trivializa toda teoría clásica que adopte un mecanismo de autorreferencia fuerte.
\end{abstract}

\section{There is (some) truth in that}

\begin{abstract}
In this paper I present a formal treatment for the notion "there is (some) truth in that". I adopt a first order language and assume a bivalued interpretation. I claim that "there is (some) truth in $X$ " behaves as a partial and not transparent truth attribution. I argue that it should be modeled using a predicate rather than an operator. I introduce a predicate and consider three alternative criteria to characterize its semantics. I prove that, with any of the criteria, the predicate trivializes any classical theory that adopts a strong self-referential procedure.
\end{abstract}

\section{Introducción}

La atribución de verdad simple, " $x$ es verdadero", es traducida al lenguaje formal mediante un predicado que se aplica a nombres de oraciones. Digamos, $T$. Hay consenso en que nuestro uso intuitivo de 'verdad' implica que $T$ debe ser 'transparente' (Cfr. Barrio, 2014: 10). Si $A$ es una oración y ${ }^{\top} A^{\top}$ ' es un nombre para $A$, debe darse que

\section{Palabras clave}

semántica formal predicado veritativo paradojas expresiones lógicas

\section{Keywords}

formal semantics truth predicate paradoxes logical expressions 
1. I.e. donde la oración es usada, no mencionada. Transparencia no implica que, v.g $T\left({ }^{\prime} A^{\prime}\right)$ y $T\left({ }^{\prime} T\left(A^{\prime}\right)^{\prime}\right)$ ') sean equivalentes.
(Transparencia) $\quad A$ y $T\left({ }^{r} A^{7}\right)$ son intersustituibles en todo contexto no opaco ${ }^{1}$

Sin embargo, Tarski probó que cualquier predicado que satisfaga Transparencia trivializa toda teoría clásica que sea capaz de probar resultados básicos sobre su propia sintaxis. Este resultado limitativo es conocido como 'indefinibilidad de la verdad' (Cfr. Horsten, 2011: 35).

En nuestro lenguaje natural contamos con una manera alternativa de atribuir verdad: "hay (algo de) verdad en eso". Un examen superficial revela que la expresión no colapsa con una atribución de verdad simple. Consideremos el pasaje:

Steve Jobs, el difunto cofundador de Apple, dijo una vez: "la creatividad es sólo conectar cosas". Hay verdad en eso, pero también existe otra fuente de creatividad: las ideas que simplemente aparecen en nuestras mentes (McCarthyJones).

La posición de Jobs puede formalizarse como $\forall x$ ( $(x$ es creativo $) \rightarrow(x$ es conectar cosas)). Supongamos que las expresiones "hay verdad en $x$ " $y$ " $x$ es verdadero" significan lo mismo. La opinión del comentador sería equivalente a $T\left({ }^{\ulcorner} \forall x((x\right.$ es creativo $) \rightarrow(x$ es conectar cosas $\left.))^{\top}\right) \wedge \exists x((x$ es creativo $) \wedge \sim(x$ es conectar cosas $))$. Por la Transparencia de $T$, se sigue $\exists x(x$ es conectar cosas $) \wedge \sim(x$ es conectar cosas $))$, i.e. una contradicción. Sin embargo, la opinión del comentador no parece contradictoria después de todo. Luego, las expresiones no significan lo mismo.

En este trabajo analizo, entonces, la semántica de "hay (algo de) verdad en $X$ ". Mis motivaciones son dos. Primero, determinar cuáles son sus condiciones de verdad en lenguaje natural, y así, sus diferencias respecto de la atribución de verdad simple. Segundo, establecer si es admisible en teorías clásicas o si, al igual que su pariente, padece de indefinibilidad.

Adopto un lenguaje de primer orden y asumo una interpretación bivaluada, es decir, tal que toda oración es verdadera o falsa, y ninguna oración es verdadera y falsa a la vez (Cfr. Falguera López \& Martínez Vidal, 1999: 140). Los resultados son los siguientes. (i) Hay tres criterios 'razonables' para caracterizar la semántica de "hay verdad en $X$ ". Presento el tratamiento formal de cada uno y dejo para futuras discusiones el asunto de cuál es el preferible. (ii) Con cualquiera de los criterios, la expresión se comporta como una atribución de verdad 'parcial': predicada de una oración compleja, solo implica la verdad de alguna de sus oraciones componentes; así, su semántica es no transparente. (iii) Con cualquiera de los criterios, la introducción en el lenguaje de un predicado para "hay verdad en $x$ " trivializa toda teoría clásica que adopte un mecanismo de autorreferencia fuerte.

El plan es sencillo. En la breve sección II, argumento que "hay verdad en $X$ " debe ser modelado utilizando un predicado y no un operador. En la sección III introduzco un predicado, $T^{9}$, y analizo cómo ha de ser su semántica para capturar "hay verdad en $X$ ". En la sección IV muestro los resultados limitativos para $T^{9}$. Para no cansar con detalles tediosos, detallo las pruebas en un Apéndice.

\section{Predicado, no operador}

La atribución de verdad simple, " $x$ es verdadero", tiene una importante función expresiva: nos permite realizar enunciados generales acerca de enunciados, como "todas tus declaraciones son falsas", "algunas verdades son incognoscibles" o "todos los teoremas son verdaderos" (Cfr. Piccolo y Schindler 2018). 
Es evidente que "hay verdad en $x$ " habilita enunciados generales también: decimos que "hay verdad en todas las tautologías", "no hay verdad en ningún juicio moral" o "hay verdad en algunas oraciones falsas". ${ }^{2}$ Es deseable que nuestra traducción al lenguaje formal preserve esta función expresiva.

A efectos de formalizar, tenemos dos opciones: usar un predicado o un operador. Un predicado se aplica a uno o más términos del lenguaje y devuelve una fórmula atómica. Un operador se aplica a una o más fórmulas y devuelve una fórmula compleja. Un término representa una frase nominal singular del lenguaje natural; una fórmula, una oración bien formada (Cfr. Boolos, 2007: 103).

Para expresar enunciados generales, necesitamos cuantificadores. Supongamos que queremos formalizar "hay verdad en todas las tautologías". Tendremos una oración del tipo

$$
\forall x(\text { Tautología }(x) \rightarrow \text { Hay-verdad-en }(x))
$$

¿Qué categoría gramatical le corresponde a la expresión ‘Hay-verdad-en’? La variable $x$ asume el lugar de un término de individuo. Los operadores no se aplican sobre términos. Luego, 'Hay-verdad-en' debe ser un predicado.

Concluimos que, como "hay verdad en $x$ " nos permite realizar enunciados generales acerca de enunciados, y queremos preservar esa función expresiva al formalizar, debemos utilizar un predicado, no un operador. ${ }^{3}$

\section{La semántica de la verdad parcial}

Sea $L_{B}$ un lenguaje de primer orden, nuestro 'lenguaje base'. Sea $L_{T^{\exists}}$ el resultado de expandir $L_{B}$ con un símbolo de predicado $T^{g}(x)$. Queremos que $T^{g}(x)$ se aplique a nombres de oraciones. Por eso, asumimos que $L_{T^{\exists}}$ contiene una constante ${ }^{r} a^{7}$ que denota $a$ para cada expresión $a \in L_{T^{\exists}}$. Se sigue que para cada oración $\varphi$ existe una constante ${ }^{\ulcorner} \varphi^{\top}$ y una oración $\left.T^{g}\left({ }^{\ulcorner} \varphi^{\top}\right)\right)$.

Sea una teoría $\Gamma \subseteq L_{T^{\exists}}$. En esta sección analizamos qué restricciones semánticas debe cumplir $\Gamma$ para que $T^{9}(x)$ signifique "hay verdad en $x$ ". Procedo por inducción sobre la posible forma lógica de $x$. En cada caso, analizo las condiciones de verdad intuitivas de "hay verdad en $X$ ", y las capturo mediante cláusulas para $T^{9}$.

Aclaración notacional: uso el alfabeto griego $\left(\varphi, \psi\right.$, etc.) para expresiones de $L_{T^{\exists}}$. Uso el alfabeto latino $(A, B$, etc.) para expresiones de nuestro lenguaje informal o natural.

\section{III.1. Oraciones atómicas}

En contextos bivaluados, la verdad se da a todo o nada, y no por grados. Luego, "hay verdad en $x$ " no significa que $x$ tenga un cierto 'grado' de verdad.

Por otro lado, una oración atómica carece de partes. Es imposible, entonces, que una 'parte' de la oración sea verdadera, pero otra 'parte' sea falsa.

Dadas estas consideraciones, podemos concluir que hay verdad en una oración atómica $A$ si y solo si $A$ es verdadera sin más. Es decir: si $\varphi \in L_{T^{\exists}}$ es atómica, entonces, para toda valuación $v, 4$
2. Estos enunciados no significan, claro está, lo mismo que sus eventuales contrapartidas formuladas con verdad simple. (Vamos a ver que un lógico clásico debe aceptar que " $\mathrm{iHay}$ verdad en todas las contradicciones!"). Sin embargo, no dejan de ser perfectamente generales.

3. No solo la verdad permite realizar generalizaciones acerca de enunciados. Halbach, Leitgeb y Welch (2003: 180) argumentan que también la necesidad debería ser entendida como un predicado, para así poder formalizar expresiones del tipo «todas las tautologías son necesarias». Alegatos similares pueden hacers respecto de otras nociones modales,

$$
v\left(T^{9}\left({ }^{\prime} \varphi^{\prime}\right)\right)=v\left(T\left({ }^{(} \varphi^{\top}\right)\right)
$$


Ahora bien: la semántica intuitiva de $T$ satisface Transparencia. Se sigue que hay verdad en una oración atómica $A$ si y solo si $A$ es el caso. Formalmente,

$\left(T^{9} \mathrm{AT}\right)$

$$
v\left(T^{g}\left({ }^{\top} \varphi^{\top}\right)\right)=v(\varphi)
$$

Vale mencionar que $\varphi$ puede ser de la forma $T^{g}\left({ }^{\top} \psi^{\top}\right)$, de modo que la cláusula ( $\left.T^{\mathrm{g}}-\mathrm{AT}\right)$ implica la equivalencia $v\left(T^{9}\left({ }^{(} \psi^{\prime}\right)\right)=v\left(T^{g}\left({ }^{(} T^{g}\left({ }^{(} \psi^{\prime}\right)^{\prime}\right)\right)$. Esto podría despertar dudas en la lectora, porque $\psi$ puede ser compleja. Sin embargo, la equivalencia resulta intuitiva. Supongamos que "hay verdad en $A$ " es falso. Luego, parece que "hay verdad en que hay verdad en $A$ " ha de ser falso también. Supongamos, ahora, que "hay verdad en $A$ " es verdadero. Entonces, parece que "hay verdad en que hay verdad en $A$ " ha de ser verdadero también. Así, nuestras intuiciones respaldan dos enunciados condicionales: si $v\left(T^{g}\left({ }^{\top} \varphi^{\top}\right)\right)=0$, entonces $v\left(T^{9}\right.$ $\left.\left({ }^{(} T^{g}\left({ }^{\top} \varphi^{\top}\right)^{\prime}\right)\right)=0$, y, si $v\left(T^{g}\left({ }^{(} \varphi^{\top}\right)\right)=1$, entonces $v\left(T^{g}\left({ }^{(} T^{g}\left({ }^{\top}\left(\varphi^{\top}\right)^{\top}\right)\right)=1\right.$. Juntos, implican que $v\left(T^{g}\left({ }^{\top} \varphi^{\top}\right)\right)=1$ si y solo si $v\left(T^{g}\left({ }^{(} T^{g}\left({ }^{(}\left(\varphi^{\top}\right)^{\top}\right)\right)=1\right.$. En otras palabras, $v\left(T^{g}\left({ }^{\top} \varphi^{\top}\right)\right)=v\left(T^{g}\left({ }^{(} T^{g}\left({ }^{\top} \varphi^{\top}\right)^{\top}\right)\right)$.

\section{III.2. Conjunciones y disyunciones}

Examinemos el siguiente diálogo, como caso paradigmático de "hay verdad en eso" en lenguaje natural:

-Creo que el alto índice de criminalidad se debe a la mala educación, a la pobreza, y a un sistema de penas demasiado indulgente.

-Hay verdad en lo que decís. La educación deficiente y la pobreza sin duda conducen a la delincuencia. Pero la severidad o no del sistema de penas no me parece un factor relevante. Hay estados que todavía tienen pena de muerte, y un índice de criminalidad peor que el nuestro.

El primer interlocutor afirma una conjunción triádica. El segundo responde que hay verdad en la conjunción, porque dos de sus coyuntos son verdaderos, y al mismo tiempo la conjunción es falsa, porque uno de los coyuntos es falso. El ejemplo justifica la idea que adelanté en la Introducción: "hay verdad en $x$ " se comporta como una atribución de verdad parcial: aplicada a una oración compleja, solo implica la verdad de alguna de sus oraciones componentes. Puede darse que una oración 'contiene' verdad, y sin embargo es en sí misma falsa. Así concebida, su semántica es por supuesto no transparente.

La pregunta que surge, ahora, es cómo tornar precisa la idea de verdad parcial. Aquí es donde entran en juego los tres criterios que anuncié:

Criterio (1): Hay verdad en una conjunción (/disyunción) si y solo si algún coyunto (/disyunto) es verdadero.

Criterio (2): Hay verdad en una conjunción (/disyunción) si y solo si hay verdad en algún coyunto (/disyunto).

Criterio (3): Hay verdad en una conjunción si y solo si algún coyunto es verdadero. Hay verdad en una disyunción si y solo si hay verdad en algún disyunto.

Vale la pena hacer algunos comentarios:

1. Si se asume Lógica Clásica, los tres criterios implican que hay verdad en toda contradicción " $A$ y no- $A$ ". La razón técnica es que, por el principio de Tercero Excluido ( $\vDash$ $A \vee \sim A$ ), uno de los coyuntos es siempre verdadero. Se puede agregar una justificación intuitiva: las contradicciones son siempre falsas porque contienen un 'exceso' de información; este mismo exceso de información es el que garantiza su verdad parcial. 
2. Una definición para "hay verdad en $X$ " es recursiva si, para determinar la verdad parcial de $A$, es suficiente con determinar la verdad parcial de cada una de sus subfórmulas. El criterio (2) permite una definición recursiva de "hay verdad en $X$ ". No ocurre lo mismo para los criterios (1) y (3), en donde la verdad parcial de una conjunción depende de la verdad 'sin más' de sus coyuntos.

3. Los criterios (1) y (3) son sensibles a la aridad de las conjunciones: hay verdad en la triádica " $\perp y A$ y no- $A$ ", pero no en la diádica " $\perp y(A y$ no- $A)$ ". El Criterio (2) es ciego en este aspecto, porque evalúa la verdad parcial de cada coyunto; así, arroja que hay verdad en ambas.

4. Con el Criterio (2), la 'verdad parcial' está cerrada bajo adición de información conjuntiva y disyuntiva: si hay verdad en " $A$ ", hay verdad en " $A$ o $B$ ", " $A$ y $B$ ", " $A$ 。 $C)$ y $B$ ", etc. Con el Criterio (3), la verdad parcial está cerrada sólo bajo adición de información disyuntiva. Con el Criterio (1), no hay ningún tipo de clausura. Para ilustrar estas diferencias: la oración " $(A$ y no- $A)$ y $(B$ y no- $B)$ " tiene verdad parcial para el Criterio (2), pero no para los criterios (1) y (3). La oración “( $A$ y no- $A)$ o (By no- $B$ )" tiene verdad parcial para los criterios (2) y (3), pero no para el Criterio (1).

Creo que los tres criterios son 'razonables', en el sentido de que (i) implican que " $A$ contiene verdad" sólo si alguna subfórmula (no negada) de $A$ es verdadera, y (ii) admiten que $A$ puede contener verdad y, al mismo tiempo, ser falsa. De esta manera, capturan nuestra idea de "hay verdad en $X$ " como 'verdad parcial'. Por el momento no encontré motivos de peso para descartar alguno de los criterios. Tampoco razones decisivas para decir que hay uno que es el 'correcto' al modelar nuestros usos lingüísticos. Como este es un trabajo exploratorio, presento el tratamiento formal de cada uno y dejo para futuras discusiones el asunto de cuál es el preferible.

Vamos a las cláusulas. Se podría pensar que para capturar el Criterio (1) basta la simple

$$
v\left(T^{g}\left({ }^{(} \varphi \wedge \psi^{\top}\right)\right)=v\left(T^{g}\left({ }^{\ulcorner} \varphi \vee \psi^{\top}\right)\right)=v(\varphi \vee \psi)
$$

Sin embargo, la cláusula es incorrecta. El operador ' $\wedge$ ', por su aridad, solo captura el Criterio (1) para conjunciones diádicas. Ejemplo: sea la triádica " $\perp$ y $A$ y no- $A$ ". Sin importar cómo distribuimos los paréntesis al formalizar, la cláusula arroja que no hay verdad en la conjunción. Pero el Criterio (1) dice lo contrario.

Para resolver el problema, lo mejor es enriquecer el lenguaje. Consideremos el lenguaje $L_{T^{\exists}}$ extendido con dos conjuntos de operadores: $\left\{\Lambda_{3}, \wedge_{4}, \ldots\right\}, y\left\{v_{3^{\prime}}, v_{4^{\prime}}, \ldots\right\}$. Ahora sí, podemos definir

$$
(\wedge, \mathrm{V}-(1)) \quad v\left(T^{g}\left({ }^{\wedge} \wedge_{\mathrm{n}}\left(\varphi_{1}, \ldots, \varphi_{\mathrm{n}}\right)^{\prime}\right)\right)=v\left(T^{g}\left({ }^{(} \mathrm{V}_{\mathrm{n}}\left(\varphi_{1}, \ldots, \varphi_{\mathrm{n}}\right)^{\prime}\right)\right)=v\left(\mathrm{~V}_{\mathrm{n}}\left(\varphi_{1}, \ldots, \varphi_{\mathrm{n}}\right)\right)
$$

Ahora el Criterio (2). Aquí, la solución es más inmediata:

$$
(\wedge, \vee-(2)) \quad v\left(T^{9}\left({ }^{(} \varphi \wedge \psi^{\top}\right)\right)=v\left(T^{9}\left({ }^{\ulcorner} \varphi \vee \psi^{\top}\right)\right)=v\left(T^{9}\left({ }^{(} \varphi^{\top}\right) \vee T^{g}\left({ }^{\top} \psi^{\top}\right)\right)
$$

Por último, el Criterio (3) combina los anteriores:

$$
\begin{aligned}
& \text { (^-(3) }) \quad v\left(T^{g}\left({ }^{\wedge} \wedge_{\mathrm{n}}\left(\varphi_{1}, \ldots, \varphi_{\mathrm{n}}\right)^{\prime}\right)\right)=v\left(\vee_{\mathrm{n}}\left(\varphi_{1}, \ldots, \varphi_{\mathrm{n}}\right)\right) \\
& \text { ( } \vee \text {-(3) } \quad v\left(T^{g}\left({ }^{\ulcorner} \varphi \vee \psi^{\top}\right)\right)=v\left(T^{9}\left({ }^{\top} \varphi^{\top}\right) \vee T^{g}\left({ }^{\ulcorner} \psi^{\top}\right)\right)
\end{aligned}
$$


5. Si $\phi$ carece de una variable $x$ libre, la cuantificación es vacua, y para toda $v, v(\forall \times(\phi))=v(\exists x(\varphi))=v(\phi)$.
6. Agradezco a un evaluador anónimo la sugerencia.

\section{III.3. Cuantificadores}

Hay consenso en que una oración universal, $\forall x(\varphi)$, expresa una conjunción infinita: " $\varphi$ se cumple cuando $x$ denota $c_{1}, y \varphi$ se cumple cuando $x$ denota $c_{2}, y . .$. " para cada $c_{i}$ en el dominio de cuantificación. Por su parte, una oración existencial, $\exists x(\varphi)$, expresa una disyunción: " $\varphi$ se cumple cuando $x$ denota $c_{1}, \circ \varphi$ se cumple cuando $x$ denota $c_{2}, 0$..." (Cfr. Falguera López \& Martínez Vidal, 1999: 274 y ss.). ${ }^{5}$

Parece razonable, entonces, extender las condiciones de 'verdad parcial' de la conjunción y la disyunción a los respectivos casos de los cuantificadores universal y existencial. Las cláusulas resultantes para los tres criterios son:

$$
\begin{aligned}
& (\forall, \exists-(1)) \quad v\left(T^{9}\left({ }^{\prime} \forall x(\varphi)^{\top}\right)\right)=v\left(T^{9}\left({ }^{\ulcorner} \exists x(\varphi)^{\top}\right)\right)=v(\exists x(\varphi)) \\
& \left(\forall, \exists-(2) \quad v\left(T^{9}\left({ }^{(} \forall X(\varphi)^{\top}\right)\right)=v\left(T^{9}\left({ }^{\ulcorner} \exists X(\varphi)^{\top}\right)\right)=v\left(\exists X\left(T^{9}\left({ }^{\ulcorner} \varphi^{\top}\right)\right)\right)\right. \\
& (\forall-3) \quad v\left(T^{g}\left({ }^{r} \forall x(\varphi)^{\top}\right)\right)=v(\exists x(\varphi)) \\
& \text { (ヨ-3) } \quad v\left(T^{9}\left({ }^{\prime} \exists X(\varphi)^{\top}\right)\right)=v\left(\exists X\left(T^{9}\left({ }^{\ulcorner} \varphi^{\top}\right)\right)\right)
\end{aligned}
$$

(En términos coloquiales: dadas las oraciones $\forall x(\varphi)$ y $\exists x(\varphi)$, (1) dice que hay verdad en ambas si y solo alguna instancia de $\varphi$ es verdadera, (2) dice que hay verdad en ambas si y solo si hay verdad en alguna instancia de $\varphi$, y (3) combina ambos criterios.)

Sin embargo, las cláusulas no son del todo satisfactorias: conducen a resultados contraintuitivos. Sea el enunciado "todos los sapos son peces". Lo formalizamos como $\forall x(\operatorname{Sapo}(x) \rightarrow \operatorname{Pez}(x))$. Si en nuestro universo existe al menos un pez, o al menos un objeto que no es un sapo, se sigue que hay verdad en el enunciado, porque es verdadero que $\exists x(\operatorname{Sapo}(x) \rightarrow P e z(x))$. El problema nos remite a la famosa 'paradoja de los cuervos' analizada por Hempel: casi todas las proposiciones categóricas universales (i.e. enunciados del tipo "Todos los $S$ son $P$ ") cuentan con instancias de confirmación 'trivial' (Cfr. Hempel, 1966: 120 y ss.). Es por esas instancias de confirmación que las cláusulas (1), (2) y (3) dicen que 'hay verdad' en tales proposiciones.

Para enfrentar el problema podemos, otra vez, enriquecer el lenguaje. ${ }^{6}$ Extendemos $L_{T^{\exists}}$ con cuantificadores acotados, de la forma $\forall x: \varphi x$ y $\exists x: \varphi x$. Estipulamos que, para tales cuantificadores, en el rango de $x$ solo hay objetos que satisfacen la fórmula $\varphi$. Ahora, formalizamos "todos los sapos son peces" como $\forall x: \operatorname{Sapo}(x)(\operatorname{Pez}(x))$. Ni la existencia de un pez en el universo, ni la existencia de algo que no es un sapo, implican $\exists x: \operatorname{Sapo}(x)(\operatorname{Pez}(x))$. Luego, podemos definir las siguientes cláusulas:

$$
\begin{array}{ll}
\left(\forall-(1)^{\prime}(3)^{\prime}\right) & v\left(T^{9}\left({ }^{(} \forall x: \varphi x(\psi)^{\prime}\right)\right)=v(\exists x: \varphi x(\psi)) \\
\left(\forall-(2)^{\prime}\right) & v\left(T^{g}\left({ }^{\prime} \forall x: \varphi x(\psi)^{\prime}\right)\right)=v\left(\exists x: \varphi x\left(T^{g}\left({ }^{\top} \Psi \Psi^{\prime}\right)\right)\right)
\end{array}
$$

en donde ninguna implica que haya verdad en oraciones como "todos los sapos son peces".

Surgen, no obstante, otros inconvenientes. Las nuevas cláusulas parecen comportarse bien cuando existe al menos una $x$ tal que $\varphi x$. Pero cuando tal $x$ no existe, son demasiado restrictivas. Supongamos que en nuestro universo no hay unicornios: se sigue que no hay verdad en "todos los unicornios son idénticos a sí mismos", lo cual es contraintuitivo. Podemos intentar salvar la situación introduciendo condicionales:

$$
\begin{aligned}
& \left(\forall-(1)^{\prime(3) ") ~} \quad v\left(T^{9}\left({ }^{(} \forall x: \varphi x(\psi)^{\top}\right)\right)=v(\exists x(\varphi x) \rightarrow \exists x: \varphi x(\psi))\right. \\
& (\forall-(2) ") \quad v\left(T^{g}\left({ }^{(} \forall x: \varphi x(\psi)^{\top}\right)\right)=v\left(\exists x(\varphi x) \rightarrow \exists x: \varphi x\left(T^{g}\left({ }^{(} \psi^{\top}\right)\right)\right)
\end{aligned}
$$


Pero no iremos muy lejos. Estas cláusulas también parecen comportarse bien cuando existe una $x$ tal que $\varphi x$. Pero cuando tal $x$ no existe, son demasiado permisivas. Dado el universo sin unicornios, implican que hay verdad en "todos los unicornios son noidénticos a sí mismos", lo cual es más contraintuitivo aún.

¿Qué concluimos entonces? Si formalizamos proposiciones categóricas universales por medio de cuantificadores no acotados, evitamos paradojas relacionadas con la inexistencia de objetos, pero sufrimos instancias de confirmación trivial. A la inversa: si usamos cuantificadores acotados, evitamos instancias de confirmación trivial, pero sufrimos paradojas de inexistencia. Así, ningún conjunto de cláusulas parece evitar casos contraintuitivos.

Sin embargo, considero que la semántica ofrecida es aceptable. Si nos comprometemos con la idea de que un cuantificador universal expresa una conjunción, y uno existencial una disyunción, entonces las cláusulas analizadas son todas correctas, en el sentido de que se limitan a extender, al nivel de las conjunciones infinitas, los criterios ofrecidos para conjunciones $n$-ádicas (apartado III.2). Estos criterios, por su parte, capturan lo que sostuve que es la característica central de "hay verdad en $X$ ": ser una atribución de verdad parcial. Luego, la causa probable de las paradojas no radica tanto en una mala especificación de las cláusulas para $T^{\natural}$, sino en que nuestros lenguajes de primer orden no capturan por completo la riqueza semántica de ciertas expresiones -como, v.g. "Todos los $S$ son $P$ "-.

En conclusión: (i) para oraciones como "todo $x$ tiene un sucesor" o "todo $x$ es distinto sí mismo", las cláusulas con cuantificadores no acotados funcionan; (ii) para oraciones como "los canguros son mamíferos" o "los mamíferos son triángulos" ("Todo $S$ es $P$ ", con la clase $S$ no vacía), los cuantificadores acotados hacen el trabajo. Por último (iii) para oraciones como "los unicornios son alados" "Todo $S$ es $P$ ", con la clase $S$ vacía) no propuse todavía cláusulas satisfactorias. Es probable que para capturar la verdad parcial de tales oraciones sea necesario apelar a enfoques intensionales, en donde la verdad de algunas oraciones no se determina (solo) por el estado de cosas en el mundo actual.

\section{III.4. Negaciones}

Sea una oración del tipo "hay (algo de) verdad en no- $A$ ".

Supongamos que $A$ es atómica. Entonces, razonamos de manera similar a lo expuesto en III.1. Apelamos a (i) el carácter 'a todo o nada' de la verdad en contextos bivaluados, y a (ii) la simplicidad de las oraciones atómicas, para concluir que hay verdad en no- $A$ si y solo si no- $A$ es verdadera. Dado que la 'verdad sin más' es transparente, se sigue también que hay verdad en no- $A$ si y solo si no- $A$ es el caso. La cláusula para $T^{9}$ es:

$$
\left(T^{\square} \sim\right) \quad \text { Si } \varphi \text { es atómica, } v\left(T^{\square}\left(\left\ulcorner\sim \varphi^{\urcorner}\right)\right)=v(\sim \varphi)\right.
$$

Como $\varphi$ puede ser de la forma $T^{9}\left({ }^{\top} \psi^{\top}\right)$, la cláusula implica la equivalencia $v\left(\sim T^{9}\left({ }^{\top} \psi^{\top}\right)\right)$ $=v\left(T^{\mathrm{g}}\left({ }^{(} \sim T^{\mathrm{g}}\left({ }^{(} \psi^{\top}\right)^{\top}\right)\right)$. La justificación intuitiva procede con un razonamiento análogo al esbozado en III.1.

Supongamos ahora que $A$ es compleja. Sea cual sea su forma lógica, podemos transformar no- $A$ en una oración intuitiva y lógicamente equivalente que nos permite deshacernos de la negación:

1. "Hay verdad en "no-no- $B$ "' equivale, por eliminación de la negación, a "Hay verdad en " $B$ "'". 
2. "Hay verdad en "no- $(B$ o $C)$ "' equivale, por De Morgan, a "Hay verdad en "no- $B$ y no- $C^{\prime \prime \prime \prime}$.

3. "Hay verdad en "no-( $B$ y $C)$ "'” equivale, por De Morgan, a "Hay verdad en "no- $B$ o no- $C^{\prime \prime \prime \prime}$.

4. "Hay verdad en "no existe un $x$ tal que $B^{\prime \prime \prime}$ equivale, por definición de los cuantificadores, a "Hay verdad en "para todo $x$, no- $B$ "'".

5. "Hay verdad en "no para todo $x, B$ "" equivale, por definición de los cuantificadores, a "Hay verdad en "existe un $x$ tal que no- $B$ "'.

Para ser riguroso, debería expresar las definiciones en forma de cláusulas para $T^{9}$. Como ninguna reviste mayor complejidad conceptual, me permito, por cuestiones de legibilidad, omitir ese paso.

Vale una aclaración. Para el predicado de verdad simple, $T$, resulta intuitiva la conmutatividad de $T$ con la negación, i.e. la equivalencia $v\left(\sim T\left({ }^{\ulcorner} A^{\top}\right)\right)=v\left(T\left({ }^{\ulcorner} \sim A^{\top}\right)\right)$. ¿Pasa lo mismo para la verdad parcial? La respuesta es negativa. Sea una contradicción " $A$ y no- $A$ ". "Hay verdad en "no- $(A$ y no- $A)$ "'" es verdadera. Pero "no hay verdad en " $A$ no- $A$ "" es falsa. Así, las expresiones "hay verdad en no- $X$ " $y$ "no hay verdad en $X$ " son no equivalentes.

Hemos caracterizado la semántica de "hay verdad en $X$ " caso por caso para cada forma lógica que puede adquirir $x$. En el apartado siguiente hago un sumario de los resultados.

\section{III.5. La semántica de la verdad parcial}

Sea una teoría $\Gamma$ formulada en $L_{T^{\exists}}$. Para capturar la noción de 'verdad parcial', o la expresión "hay (algo de) verdad en $X$ ", $\Gamma$ debe satisfacer la siguiente semántica, para uno de los criterios(1), (2) o (3):

$$
\begin{aligned}
& \left(T^{g} \mathrm{At}\right) \quad \text { Si } \varphi \text { es atómica, } v\left(T^{9}\left({ }^{\ulcorner} \varphi^{\top}\right)\right)=v(\varphi) \\
& \left(T^{9} \sim\right) \quad \text { Si } \varphi \text { es atómica, } v\left(T^{9}\left(\left\ulcorner\sim \varphi^{\top}\right)\right)=v(\sim \varphi)\right. \\
& (\wedge, \vee-1) \quad v\left(T^{g}\left({ }^{\ulcorner} \wedge_{\mathrm{n}}\left(\varphi_{1}, \ldots, \varphi_{\mathrm{n}}\right)^{\top}\right)\right)=v\left(T^{\exists}\left({ }^{\ulcorner} \vee_{\mathrm{n}}\left(\varphi_{1}, \ldots, \varphi_{\mathrm{n}}\right)^{\top}\right)\right)=v\left(\vee_{\mathrm{n}}\left(\varphi_{1}, \ldots, \varphi_{\mathrm{n}}\right)\right) \\
& (\forall, \exists-(1)) \quad v\left(T^{g}\left({ }^{\ulcorner} \forall x(\psi)^{\top}\right)\right)=v\left(T^{g}\left({ }^{\ulcorner} \exists x(\varphi)^{\top}\right)\right)=v(\exists x(\psi)) \\
& \left(\forall-(1)^{\prime}\right) \quad v\left(T^{g}\left({ }^{\ulcorner} \forall x: \varphi x(\psi)^{\top}\right)\right)=v(\exists x: \varphi x(\psi)) \\
& (\wedge, \vee-(2)) \quad v\left(T^{g}\left({ }^{\ulcorner} \varphi \wedge \psi^{\urcorner}\right)\right)=v\left(T^{g}\left(\left\ulcorner\varphi \vee \psi^{\top}\right)\right)=v\left(T^{g}\left({ }^{\ulcorner} \varphi^{\top}\right) \vee T^{g}\left({ }^{\ulcorner} \psi^{\urcorner}\right)\right)\right. \\
& (\forall, \exists-(2)) \quad v\left(T^{9}\left({ }^{\ulcorner} \forall x(\psi)^{\top}\right)\right)=v\left(T^{g}\left({ }^{\top} \exists x(\psi)^{\urcorner}\right)\right)=v\left(\exists x\left(T^{9}\left({ }^{\ulcorner} \psi^{\urcorner}\right)\right)\right) \\
& \left(\forall-(2)^{\prime}\right) \quad \quad \quad v\left(T^{g}\left({ }^{(} \forall x: \varphi x(\psi)^{\urcorner}\right)\right)=v\left(\exists x: \varphi x\left(T^{g}\left({ }^{\top} \psi^{\urcorner}\right)\right)\right) \\
& \text {Criterio(3) ( }, \forall-(1)),(\vee, \exists-(2)),\left(\forall-(1)^{\prime}\right)
\end{aligned}
$$

(Como adelanté, omito detallar las cláusulas 1-5 para oraciones negadas (III.4)).

Damos por cumplido el primer objetivo de este trabajo: analizar la semántica de "hay (algo de) verdad en $X$ ". En la breve sección siguiente me aboco al segundo de los objetivos. 


\section{Indefinibilidad}

Como mencioné en la Introducción, Tarski probó que un predicado transparente trivializa toda teoría clásica capaz de probar resultados básicos sobre su propia sintaxis. Como la noción intuitiva de 'verdad' se formaliza con un predicado transparente, el resultado es conocido como 'indefinibilidad de la verdad'.

Ahora bien: el predicado $T^{9}$, que usamos para formalizar "hay verdad en eso", es no transparente. Se plantea entonces la pregunta de si $T^{9}$ evita el resultado de indefinibilidad.

Decimos que una teoría $\Gamma \subseteq L_{T^{\exists}}$ adopta un mecanismo de autorreferencia fuerte si para cada fórmula $A(x)$, con $x$ como única variable libre, existe un término $t$ tal que $\Gamma$ implica $t=A(t)$ (Cfr. Barrio, Pailos y Szmuc, 2017: 390).

Proposición 1. Sea $\Gamma \subseteq L_{T^{\exists}}$ una teoría clásica que adopta un mecanismo de autorreferencia fuerte. Si $\Gamma$ satisface la cláusula para la negación $\left(T^{9} \sim\right), \Gamma$ es inconsistente.

La PRUEBa se detalla en el Apéndice. Lo central es que existe un término mentiroso $\lambda$ tal que $\Gamma$ implica ${ }^{\Gamma} \lambda^{\top}={ }^{\Gamma} \sim T^{g}(\lambda)^{\top}$. No hay una asignación de valor estable para la oración $T^{g}(\lambda)$.

Para el Criterio (1), es posible probar un resultado aún más fuerte:

Decimos que una teoría $\Gamma \subseteq L_{T^{\exists}}$ adopta un mecanismo de autorreferencia débil si para cada fórmula $A(x)$, con $x$ como única variable libre, existe una oración $G$ tal que $\Gamma$ implica $G \leftrightarrow A\left({ }^{\ulcorner} G^{\top}\right)$.

Por el Lema Diagonal de Gödel, sabemos que la aritmética mínima Q cuenta con un mecanismo de autorreferencia débil (Cfr. Boolos, 2007: 237).

Proposición 2. Sea $\Gamma \subseteq L_{T^{\exists}}$ una teoría clásica que extiende la aritmética mínima, Q. Si $\Gamma$ satisface la cláusula del Criterio (1) para el cuantificador existencial ( $\exists-(1)), \Gamma$ es inconsistente.

La PRUEBA se detalla en el Apéndice. Lo central es que existe una oración mentirosa $\lambda$ tal que $\Gamma$ implica $\lambda \leftrightarrow \sim T^{g}\left({ }^{\prime} \lambda^{\top}\right)$. No hay una asignación de valor estable para $\lambda$.

Concluimos que, a pesar de ser no transparente, la verdad parcial enfrenta resultados limitativos emparentados a los de la 'verdad sin más'.

\section{Conclusiones}

A lo largo del trabajo analizamos la semántica de la expresión "hay (algo de) verdad en $X$ ", o atribución de verdad parcial. Adoptamos un lenguaje de primer orden y asumimos una interpretación bivaluada. Consideramos tres criterios razonables para caracterizar la semántica de "hay (algo de) verdad en $X$ ", y capturamos cada criterio por medio de cláusulas para el predicado $T^{9}$. Mostramos que $T^{9}$ trivializa toda teoría clásica que adopte mecanismos de autorreferencia fuerte.

Considero que los objetivos para indagaciones futuras son los siguientes. Primero, analizar cuál de los criterios es el preferible a la hora de modelar nuestros usos lingüísticos. Segundo, adoptar un enfoque sintáctico, y explorar los principios inferenciales que gobiernan "hay (algo de) verdad en $x$ ". Por último, extender el 
7. Las lógicas no clásicas estructurales son aquellas que rechazan algún principio inferencial válido en Lógica Clásica, como la ley de tercero excluido o el principio de explosión. Las subestructurales son aquellas que rechazan algún principio metainferencial o 'regla estructural', como transitividad o contracción. Véase Barrio

(2014) para el uso de lógicas $n$ clásicas estructurales en la formulación de teorías de la verdad transparentes. Véase, v.g. Ripley (2012) o Zardini (2011) para el uso de lógicas subestructurales con el mismo fin. tratamiento semántico a contextos multivaluados, y analizar la admisibilidad de $T^{9}$ en lógicas no clásicas, tanto estructurales como subestructurales. ${ }^{7}$

\section{Apéndice}

\section{Prueba de la Proposición 1.}

Como $\Gamma$ adopta autorreferencia fuerte, existe un término $\lambda$ tal que $\Gamma$ implica $\lambda=$ ${ }^{\ulcorner} \sim T^{g}(\lambda)^{7}$. Mostramos que no hay una asignación de valores consistente para $T^{g}(\lambda)$ :

Suponemos que $v\left(T^{g}(\lambda)\right)=1$. Por sustitución de idénticos, $v\left(T^{\mathrm{g}}\left(\sim^{\mathrm{T}} \sim T^{\mathrm{g}}(\lambda)^{\top}\right)\right)=1$. Aplicamos la cláusula $T^{g} \sim$ y obtenemos $v\left(\sim T^{g}(\lambda)\right)=1$, por lo que $v\left(T^{g}(\lambda)\right)=0$; contradicción. Concluimos, entonces, que $v\left(T^{\mathrm{g}}(\lambda)\right)=0$. Por sustitución de idénticos, $v\left(T^{\mathrm{g}}\left({ }^{(} \sim T^{\mathrm{g}}(\lambda)^{\top}\right)\right)$ $=0$. Aplicamos $T^{g} \sim$ otra vez y obtenemos $v\left(\sim T^{g}(\lambda)\right)=0$, por lo que $v\left(T^{g}(\lambda)\right)=1$; contradicción.

\section{Prueba de la Proposición 2.}

Dada una fórmula $A(x)$ con $x$ como única variable libre, su diagonalización equivale a la oración $A\left({ }^{\top} A^{\top}\right)$. Existe en $\mathrm{Q}$ una fórmula $\operatorname{Diag}(\mathrm{x}, \mathrm{y})$ tal que, si $B$ es la diagonalización $\operatorname{de} A, \mathrm{Q} \vDash \forall y\left(\operatorname{Diag}\left({ }^{\ulcorner} A^{\top}, y\right) \leftrightarrow y={ }^{\top} B^{\top}\right)$.

Por el Lema Diagonal, para cada fórmula $B(y)$ en Q existe una oración $G$ tal que Q $\vDash G \leftrightarrow B\left({ }^{\ulcorner} G^{\urcorner}\right)$. La oración $G$ es de la forma $\exists x\left(x={ }^{\ulcorner} \exists y(\operatorname{Diag}(x, y) \wedge B(y))^{\urcorner} \wedge \exists y(\operatorname{Diag}(x, y)\right.$ $\wedge B(y)))$.

Como $\mathrm{Q} \subseteq \Gamma$, existe en $\Gamma$ una oración $\lambda$

(

$$
\exists x\left(x={ }^{\ulcorner} \exists y\left(\operatorname{Diag}(x, y) \wedge \sim T^{\exists}(y)\right)^{\urcorner} \wedge \exists y\left(\operatorname{Diag}(x, y) \wedge \sim T^{\exists}(y)\right)\right)
$$

tal que $\Gamma \vDash \lambda \leftrightarrow \sim T^{g}\left({ }^{\top} \lambda^{\top}\right)$. Mostramos que no hay una asignación de valores consistente para $\lambda$ :

Suponemos que $v(\lambda)=1$. Por el Lema Diagonal, $v\left(\sim T^{g}\left({ }^{\top} \lambda\right)\right)=1$, de modo que $v\left(T^{g}\right.$ $\left.\left(\lambda^{\top}\right)\right)=0$. Como $\lambda$ es de la forma $\exists x(\varphi)$, aplicamos la cláusula $\exists-1$ y obtenemos $v(\lambda)=0$; contradicción. Concluimos, entonces, que $v(\lambda)=0$. Por el Lema Diagonal, $v\left(\sim T^{g}\left({ }^{(} \lambda^{\top}\right)\right)=0$, de modo que $v\left(T^{g}\left({ }^{\ulcorner} \lambda^{\top}\right)\right)=1$. Aplicamos $\exists$ - 1 otra vez y obtenemos $v(\lambda)=1$; contradicción. 


\section{Bibliografía}

" Barrio, E. (Dir.). (2014). La lógica de la verdad. Buenos Aires: Eudeba.

" Barrio, E., Pailos F. \& Szmuc D. (2017). A paraconsistent route to semantic closure. Logic Journal of the IGPL, 25 (4), 387-407. Doi: 10.1093/jigpal/jzx009

" Boolos, G., Burgess, J. \& Jeffrey, R. (2007). Computability and Logic. Cambridge: Cambridge University Press.

» Falguera López, J. L. \& Martínez Vidal, C. (1999). Lógica Clásica de Primer Orden: Estrategias de Deducción, Formalización y Evaluación Semántica. Madrid: Trotta.

" Halbach, V., Leitgeb, H. \& Welch, Ph. (2003). Possible-worlds semantics for modal notions conceived as predicates. Journal of Philosophical Logic, 32(2), 179-223. Doi: 10.1023/A:1023080715357.

» Hempel, C. G. (1966). Recent problems of induction. En R. Colodny (Ed.), Mind and cosmos. Essays in Contemporary Sience and Philosophy (112-134). Pittsburgh: University of Pittsburgh Press.

» Horsten, L. (2011). The Tarskian turn: Deflationism and axiomatic truth. Cambridge: Massachusetts Institute of Technology.

" McCarthy-Jones, S. Entrada en InnerSelf:<https://es.innerself.com/content/living/ leisure-and-creativity/16026-brain-stimulation-can-boost-creativity-and-maybe-hear-inspirational-voices.html> Recuperado: 24.12.2018.

"Picollo, L. \& Schindler, T. (2018). Disquotation and infinite conjunctions. Erkenn, 83(5), 899-928. Doi: 10.1007/s10670-017-9919-x.

» Ripley, D. (2012). Conservatively Extending Classical Logic with Transparent Truth. Review of Symbolic Logic, 5(2), 354-378. Doi: 10.1017/S1755020312000056.

"Zardini, E. (2011). Truth without Contra(di)ction. Review of Symbolic Logic, 4(4), 498-535. Doi: 10.1017/S1755020311000177 
